\title{
Applicability of Different Isothermal EOS at Nanomaterials
}

\author{
Deepika P. Joshi ${ }^{1}$ and Anjali Senger ${ }^{2}$ \\ ${ }^{1}$ Department of Physics, G. B. Pant University of Agriculture and Technology, Pantnagar, Uttarakhand 263145, India \\ ${ }^{2}$ Division of Physics and Applied Physics, School of Physical and Mathematical Sciences, Nanyang Technological University, Singapore
}

Correspondence should be addressed to Deepika P. Joshi; deepikakandpal@gmail.com

Received 4 April 2013; Accepted 20 May 2013

Academic Editor: Ravindra R. Pandey

Copyright (c) 2013 D. P. Joshi and A. Senger. This is an open access article distributed under the Creative Commons Attribution License, which permits unrestricted use, distribution, and reproduction in any medium, provided the original work is properly cited.

\begin{abstract}
The present study explains the behaviour of nanomaterials such as AlN, CdSe, Ge , WC , and Ni- and Fe-filled-MWCNTs under high pressure. Among the number of isothermal EOSs available, we prefer only two parameter-based isothermal equations (i.e., Murnaghan equation, usual Tait's equation, Suzuki equation and Shanker equation). The present work shows the theoretical study of thermo-elastic properties especially relative compression $\left(V / V_{0}\right)$, isothermal bulk modulus $\left(K_{P} / K_{0}\right)$, and compressibility $\left(\alpha_{(P)} / \alpha_{0}\right)$ of nanomaterials. After comparing all formulations with available experimental data, we conclude that pressure dependence of relative compression $\left(V / V_{0}\right)$ for the nanomaterials, are in good agreement for all the equations at lower pressure range. At higher pressure range, Suzuki and Shanker formulations show some deviation from experimental values.
\end{abstract}

\section{Introduction}

Nanomaterials are currently in the focus of intense research due to their potential for revolutionary technological applications in diverse areas $[1,2]$. Nanomaterials can be metals, ceramics, polymeric materials, or composite materials. Their defining characteristic is the very small feature size in the range of 1-100 nanometers (nm). Nanomaterials are not just simply another step of minimization but an entirely different arena. At the nanomaterial level, some material properties are affected by the laws of atomic physics, rather than behaving as traditional bulk materials.

Many of the mechanical properties at nanolevel are modified and different from their bulk counterpart, including the hardness, elastic modulus, fracture, toughness, scratch resistance, and fatigue strength. High hardness has been discovered in many nanomaterials system.

Nanosemiconductors with reduced dimensions recently have been shown to exhibit electronic and optical properties which vary with size of the particles, thus making them potential candidates for applications involving tenability of optical and/or electronic properties [3-5].
Tungsten carbide WC is an important nanocomposite, because of its high melting point and hardness; it is important materials in both industry and high-pressure research. WC finds extensive applications in industrial machinery as cutting tools and abrasives. WC is also widely used as anvil materials in multianvil high-pressure instruments and as seats in diamond anvil cells. Moreover, nano-WC with average grain sizes less than $100 \mathrm{~nm}$ has been the subject of active research over the past decades, primarily due to the significant roles grain-size reduction played in the enhancements of mechanical properties $[6,7]$.

There is another class of nanosystems that are quite interesting due to their unique features, that is, nanotubes and nanowires. In particular, due to the high mechanical strength and ballistic electronic conduction, carbon nanotubes are beginning to find several uses such as for scanning probes, electronic transistors, field-emitting devices, and energy storage. Nanotubes can also be filled with biological molecules, raising the possibility of applications in biotechnology. Encapsulation of various metals in multiwalled carbon nanotubes (MWCNTs) is being used to study the physical properties of nanowires and nanoparticles of these metals. Transition-metal nanowires, encapsulated inside the 
multiwalled carbon nanotubes, are promising materials for use in nanodevices and in the magnetic storage industry and for spintronics materials. Several interesting results have been obtained on filled MWCNTs, for example, Fe- and Ni-filled MWCNTs [8].

What are the expected effects or benefits of high pressures on nanomaterials? This is simply because, in addition to composition and synthetic routes, high pressure provides an additional effective driving force to produce new structures and, therefore, new nanomaterial properties. The high pressure research has truly developed an interdisciplinary area that has important applications in the field of science. The high pressure study is important not only for better understanding of matter in the world around us, but also to create entirely new form of matter. One of the most important outputs of high pressure experiment is the pressure-volumetemperature $(P, V, T)$ relationship termed as equation of state. Study of equation of state for solids has been extremely useful in the field of geophysics and condensed matter physics, with possible application in many fields. The study based on the EOS at high pressure is of fundamental interest because it permits interpolation and extrapolation into the regions for which the experimental data are not available adequately [9-18].

The purpose of the present study is to access the validity of some important and widely used EOS at high pressure on nanomaterials. We have done the study of the Murnaghan, usual Tait, Suzuki, and Shanker equations under various pressure ranges for different classes of nanomaterials and results are compared with available experimental data. The present work describes the theoretical study of thermoelastic properties especially relative compression $\left(V / V_{0}\right)$, bulk modulus $\left(K_{(P)} / K_{0}\right)$, and compressibility $\left(\alpha_{(P)} / \alpha_{0}\right)$ of AlN (10 nm), Ge $(13 \mathrm{~nm}), \mathrm{CdSe}(5.4 \mathrm{~nm}), \mathrm{Ni}$ - and Fe-filled MWCNTs, and WC $(25 \mathrm{~nm})$ nanomaterials at high pressure.

\section{Method of Analysis}

In this work, we have employed the potential-free model, which is developed by incorporating several important thermodynamical relationships $[9,19,20]$. Our main purpose of the present study is to provide a straightforward and simple method rather than considering a potential model based on several approximations to analyze the thermoelastic properties of nanomaterials. We have studied the pressure dependence of volume compression, bulk modulus, and compression coefficient of nanomaterials such as n-AlN, nGe, n-CdSe, Ni- and Fe-filled MWCNT, and n-WC using the following equations of state.

2.1. Murnaghan Equation of State. The well-known and widely used EOS [21] is the Murnaghan equation of state, which is based on the assumption that isothermal bulk modulus $K$ is a linear function of pressure at any temperature; that is

$$
K(P, T)=K_{0}+K_{0}^{\prime} P
$$

Using the definition of bulk modulus and integrating equation (1) at constant temperature, we get the Murnaghan equation of state as follows:

$$
\frac{V}{V_{0}}=\left(1+\frac{K_{0}^{\prime}}{K_{0}} P\right)^{-1 / K_{0}^{\prime}}
$$

Bulk modulus is written as

$$
\frac{K_{P}}{K_{0}}=\left(\frac{V}{V_{0}}\right)^{-K_{0}^{\prime}}
$$

Using the well-established thermodynamic approximation [22-24] that is under the effect of pressure the product of $\alpha_{(P)}$ and $K_{(P)}$ remains constant:

$$
\alpha K_{P}=\alpha_{0} K_{0}
$$

where $\alpha_{0}$ and $K_{0}$ are the values of $\alpha$ and $K$ at zero pressure. We get the expression

$$
\frac{\alpha_{P}}{\alpha_{0}}=\left(\frac{V}{V_{0}}\right)^{K_{0}^{\prime}}
$$

Equation (5) is a useful relation for predicting the pressure dependence of $\alpha_{(P)}$ along isotherm.

2.2. Usual Tait Equation of State. The usual Tait equation is most useful for nonlinear relation of compression and pressure for different class of solids and liquids [25]. Kumar $[26,27]$ presented the derivation of this equation. The usual Tait's equation (UTE) can be written as follows:

$$
\frac{V}{V_{0}}=\left[1-\frac{1}{K_{0}^{\prime}+1} \ln \left\{1+\left(\frac{K_{0}^{\prime}+1}{K_{0}}\right) P\right\}\right] .
$$

Using usual Tait's equation (UTE), the expression for isothermal bulk modulus $K_{(P)}$ is written as follows [28, 29]:

$$
\frac{K_{(P)}}{K_{(0)}}=\frac{V}{V_{0}} \exp \left\{\left(K_{0}^{\prime}+1\right)\left(1-\frac{V}{V_{0}}\right)\right\} .
$$

This is the equation for isothermal bulk modulus.

Using the well-established thermodynamic approximation [22-24] that is under the effect of pressure the product of $\alpha_{(P)}$ and $K_{(P)}$ remains constant

$$
\alpha K_{P}=\alpha_{0} K_{0}
$$

where $\alpha_{0}$ and $K_{0}$ are the values of $\alpha$ and $K$ at zero pressure, we get the expression following:

$$
\frac{\alpha_{P}}{\alpha_{0}}=\left(\frac{V}{V_{0}}\right)^{-1} \exp \left\{-\left(K_{0}^{\prime}+1\right)\left(1-\frac{V}{V_{0}}\right)\right\} \text {. }
$$

Equation (9) is the relation of the pressure dependence of $\alpha_{(P)}$ along isotherm. 
2.3. Equation of State Based on Suzuki Formulation. SanMiguel and Suzuki [18, 19] have followed the Gruneisen theory of thermal expansion based on the Mie-Gruneisen equation of state [30]:

$$
P V+X(V)=\gamma E_{\mathrm{Th}},
$$

where $P$ is pressure, $X(V)=(d \Phi / d V)$, $\Phi$ is potential energy as a function of volume only, $\gamma$ is the Gruneisen parameter regarded as constant, and $E_{\mathrm{Th}}$ is the thermal energy of lattice vibration. After applying Taylor's expansion to the second term in (10), with respect to the second order, and solving we get

$$
\frac{V}{V_{0}}=\frac{\left[1+2 k-\left(1-\left(4 k E_{\mathrm{Th}} / Q\right)\right)^{1 / 2}\right]}{2 k} .
$$

In the Mie-Gruneisen EOS,

$$
P_{\mathrm{Th}}=\frac{\gamma E_{\mathrm{Th}}}{V_{0}}
$$

since

$$
Q=\frac{K_{0} V_{0}}{\gamma} .
$$

Using (12) and (13), (11) becomes

$$
\frac{V}{V_{0}}=\frac{1+2 k-\left(1-\left(4 k P_{\mathrm{Th}} V / K_{0} V_{0}\right)\right)^{1 / 2}}{2 k} .
$$

Taking $k=\left(\left(K_{0}^{\prime}-1\right) / 2\right)$, where $K_{0}^{\prime}$ is the first pressure derivative of bulk modulus, we get

$$
\begin{gathered}
\frac{V}{V_{0}}=\frac{1+\left(K_{0}^{\prime}-1\right)-\left[1-2\left(\left(K_{0}^{\prime}-1\right) / K_{0}\right) P_{\mathrm{Th}}\right]^{1 / 2}}{\left(K_{0}^{\prime}-1\right)}, \\
\frac{V}{V_{0}}-1=\frac{1-\left[1-2\left(\left(K_{0}^{\prime}-1\right) / K_{0}\right) P_{\mathrm{Th}}\right]^{1 / 2}}{\left(K_{0}^{\prime}-1\right)},
\end{gathered}
$$

where $P_{\mathrm{Th}}$ is the thermal pressure. Following the arguments of Shanker and Kushwah [24], when $P$ is not equal to zero, (16) may be rewritten as follows:

$$
\frac{V}{V_{0}}-1=\frac{1-\left[1-2\left(\left(K_{0}^{\prime}-1\right) / K_{0}\right)\left(P-P_{\mathrm{Th}}\right)\right]^{1 / 2}}{\left(K_{0}^{\prime}-1\right)}
$$

Now, when thermal pressure is zero $\left(P_{\mathrm{Th}}\right),(17)$ gives the following simple relation:

$$
\frac{V}{V_{0}}-1=\frac{1-\left[1+2\left(\left(K_{0}^{\prime}-1\right) / K_{0}\right) P\right]^{1 / 2}}{\left(K_{0}^{\prime}-1\right)},
$$

or

$$
\begin{aligned}
& \frac{V}{V_{0}}=\frac{1-\left[1+2\left(\left(K_{0}^{\prime}-1\right) / K_{0}\right) P\right]^{1 / 2}}{\left(K_{0}^{\prime}-1\right)}+1, \\
& \frac{K_{(P)}}{K_{0}}=\left[\frac{V}{V_{0}}\left(1+\left(K_{0}^{\prime}-1\right)\left(1-\frac{V}{V_{0}}\right)\right)\right] .
\end{aligned}
$$

Using the identity $\alpha K_{P}=\alpha_{0} K_{0}$ where, $\alpha_{0}$ and $K_{0}$ are the value of $\alpha$ and $K$ at zero pressure, we get the following:

$$
\frac{\alpha_{(P)}}{\alpha_{0}}=\left[\frac{V}{V_{0}}\left(1+\left(K_{0}^{\prime}-1\right)\left(1-\frac{V}{V_{0}}\right)\right)\right]^{-1} .
$$

Equation (21) shows the variation of $\alpha_{(P)}$ with pressure at constant temperature.

2.4. Shanker Formulation. The Gruneisen theory of thermal expansion as formulated by Born and Huang has been used by Shanker et al. [31]. These authors included higher order term for the change in volume in the expansion of potential energy and claimed to derive a new expression for $V / V_{0}$ which reads as follows:

$$
\frac{V}{V_{0}}-1=\frac{1-\left[1+2\left(\left(K_{0}^{\prime}+1\right) / K_{0}\right) P_{\mathrm{Th}}\right]^{1 / 2}}{\left(K_{0}^{\prime}+1\right)} .
$$

It has been argued by Kushwaha and Shanker that the above EOS may be rewritten as follows when $P$ is not equal to zero:

$$
\frac{V}{V_{0}}-1=\frac{1-\left[1+2\left(\left(K_{0}^{\prime}+1\right) / K_{0}\right)\left(P-P_{\mathrm{Th}}\right)\right]^{1 / 2}}{\left(K_{0}^{\prime}+1\right)}
$$

When thermal pressure is zero $\left(P_{\mathrm{Th}}=0\right)(23)$ gives

$$
\frac{V}{V_{0}}-1=\frac{1-\left[1+2\left(\left(K_{0}^{\prime}+1\right) / K_{0}\right) P\right]^{1 / 2}}{\left(K_{0}^{\prime}+1\right)},
$$

or

$$
\frac{V}{V_{0}}=\frac{1-\left[1+2\left(\left(K_{0}^{\prime}+1\right) / K_{0}\right) P\right]^{1 / 2}}{\left(K_{0}^{\prime}+1\right)}+1 .
$$

Now bulk modulus

$$
\frac{K_{(P)}}{K_{0}}=\frac{V}{V_{0}}\left(1+\left(K_{0}^{\prime}+1\right)\left(1-\frac{V}{V_{0}}\right)\right) .
$$

Using the identity $\alpha K_{P}=\alpha_{0} K_{0}$ with (26) we get the following:

$$
\frac{\alpha_{(P)}}{\alpha_{0}}=\left[\frac{V}{V_{0}}\left(1+\left(K_{0}^{\prime}+1\right)\left(1-\frac{V}{V_{0}}\right)\right)\right]^{-1} .
$$

Equations (26) and (27) are useful relations for showing the pressure dependence of $K_{(P)}$ and $\alpha_{(P)}$, respectively.

\section{Results}

High pressure study of nanomaterials has been described under the Murnaghan, usual Tait, Suzuki, and Shanker formulations because of their simple and straightforward applications in high pressure physics. In this paper, we have reported the results obtained for thermophysical properties of some nanomaterials, that is, AlN (10 nm), Ge (13 nm), CdSe $(5.4 \mathrm{~nm}), \mathrm{Ni}$ - and Fe-filled MWCNTs, and WC (25 nm) under the effect of high pressure. The values of input parameters 


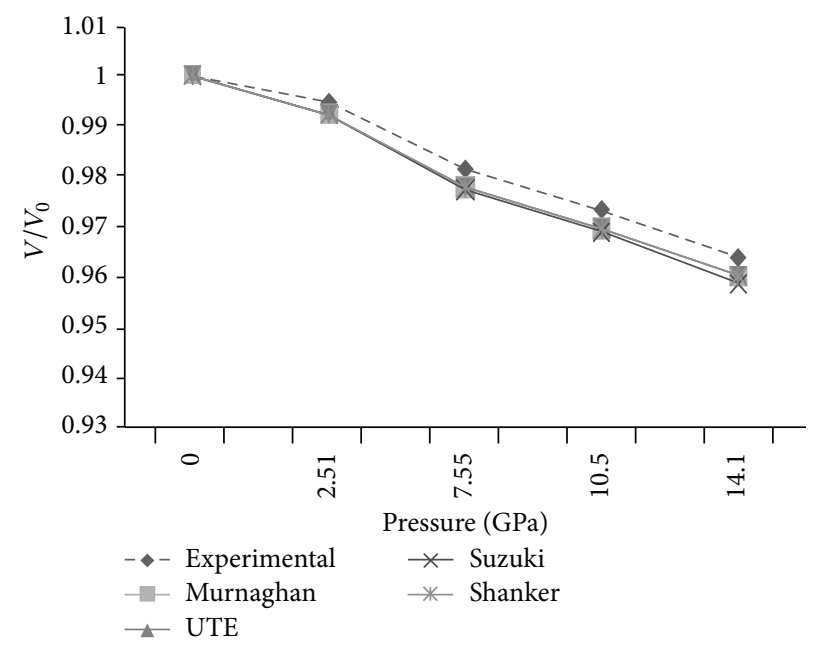

(a) $\operatorname{AlN}(10 \mathrm{~nm})$

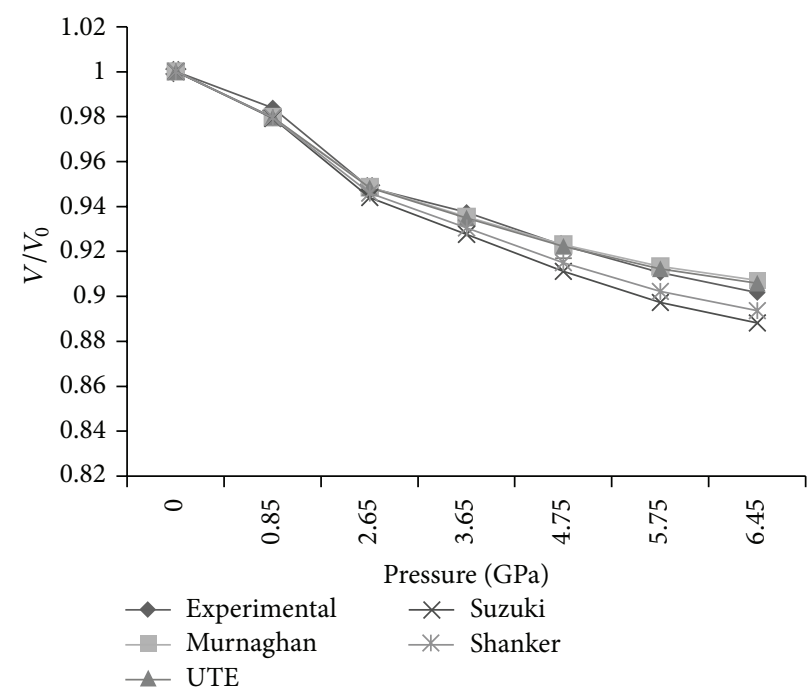

(c) CdSe $(5.2 \mathrm{~nm})$

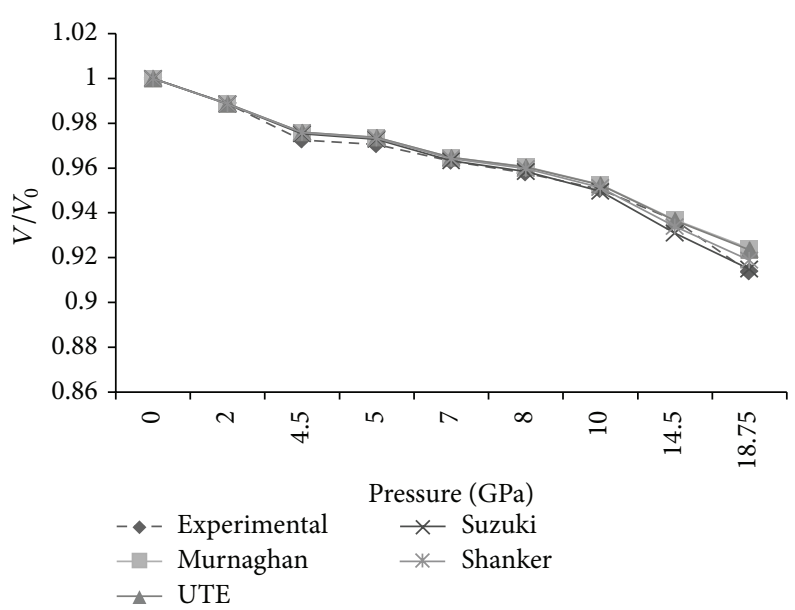

(e) Fe-filled MWCNT

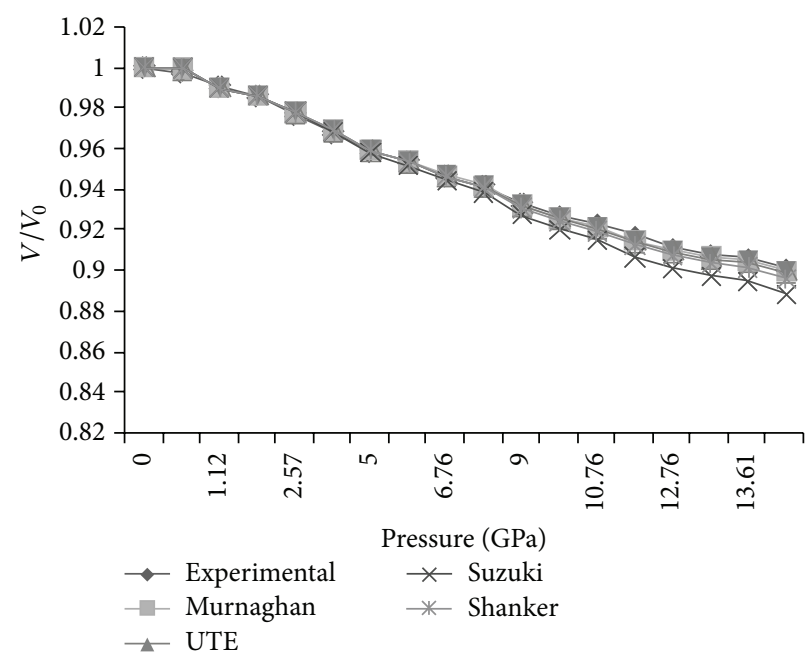

(b) $\mathrm{Ge}(13 \mathrm{~nm})$

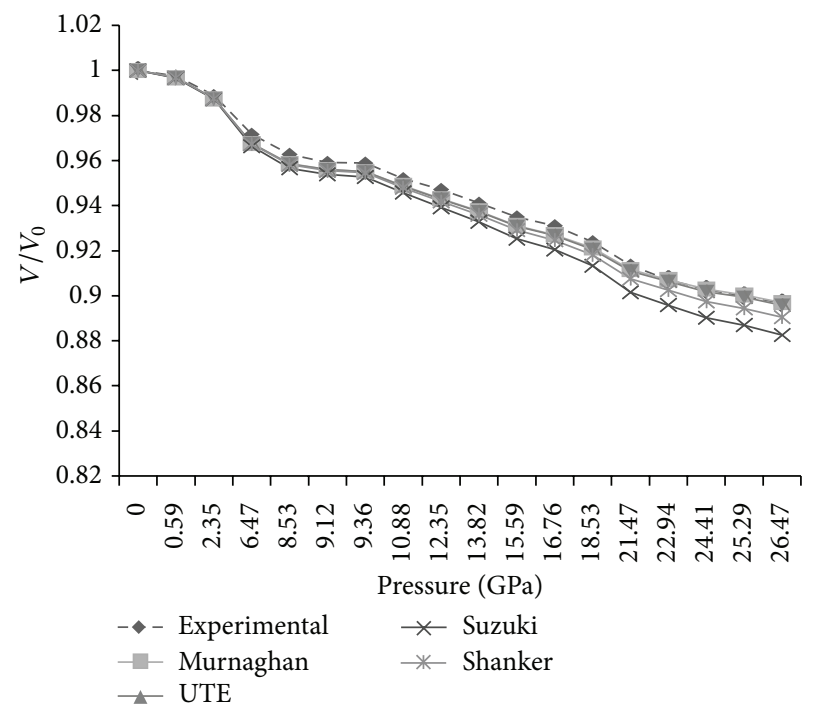

(d) Ni-filled MWCNT

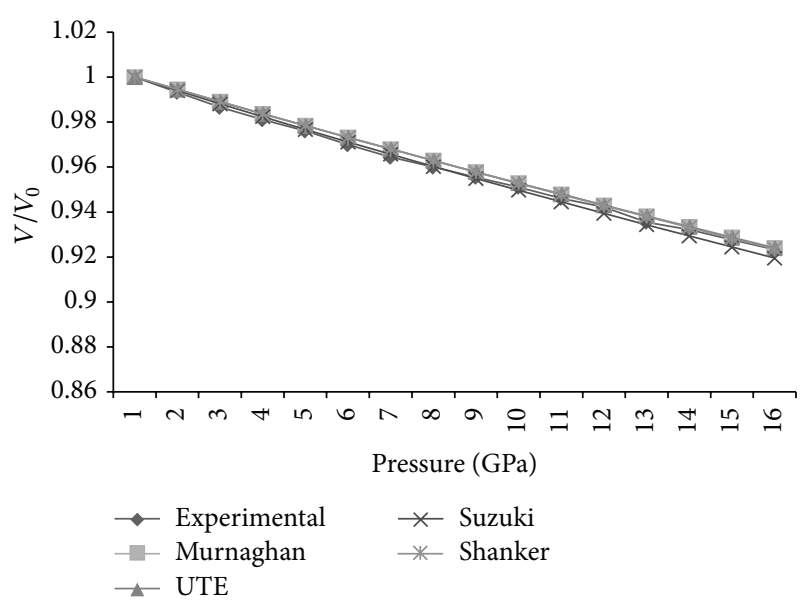

(f) $\mathrm{WC}(25 \mathrm{~nm})$

FIgURE 1: Variation of relative volume compression $\left(V / V_{0}\right)$ with pressure $(P)$ for different materials. 


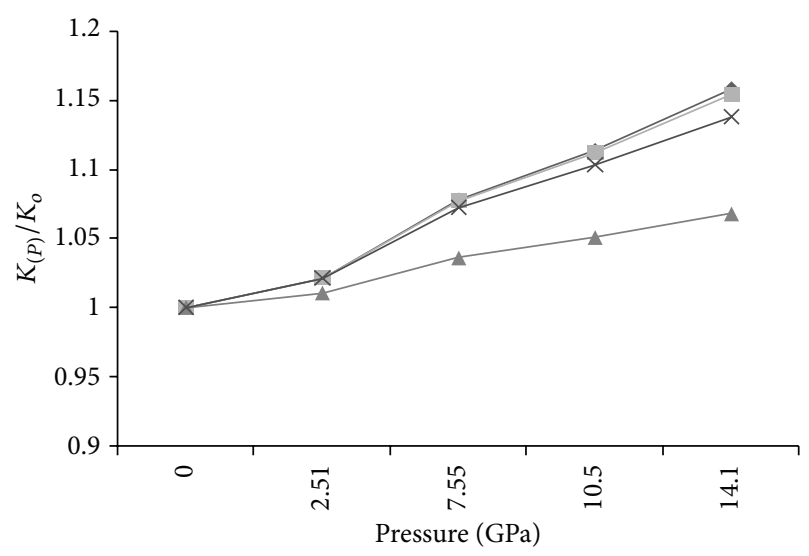

(a) $\operatorname{AlN}(10 \mathrm{~nm})$

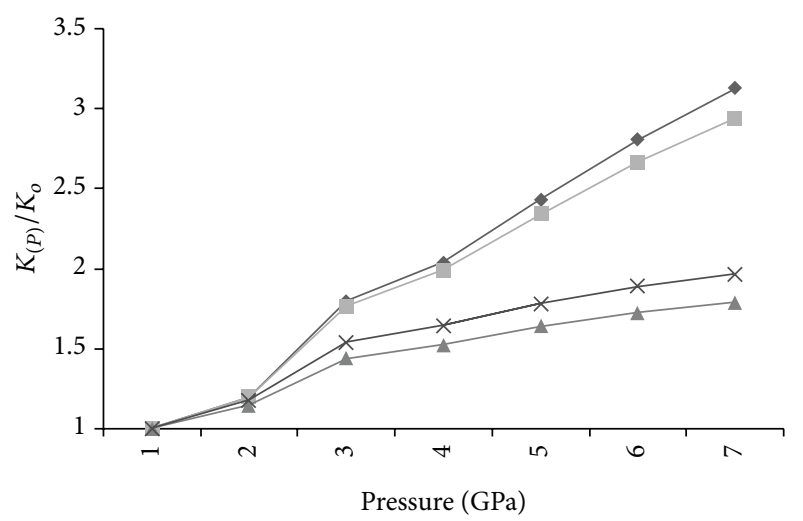

(c) $\mathrm{CdSe}(5.2 \mathrm{~nm})$

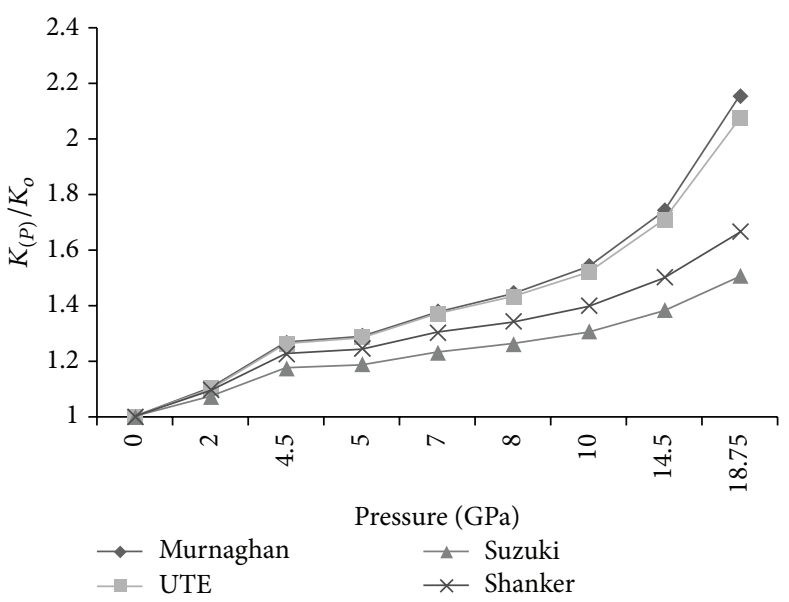

(e) Fe-filled MWCNT

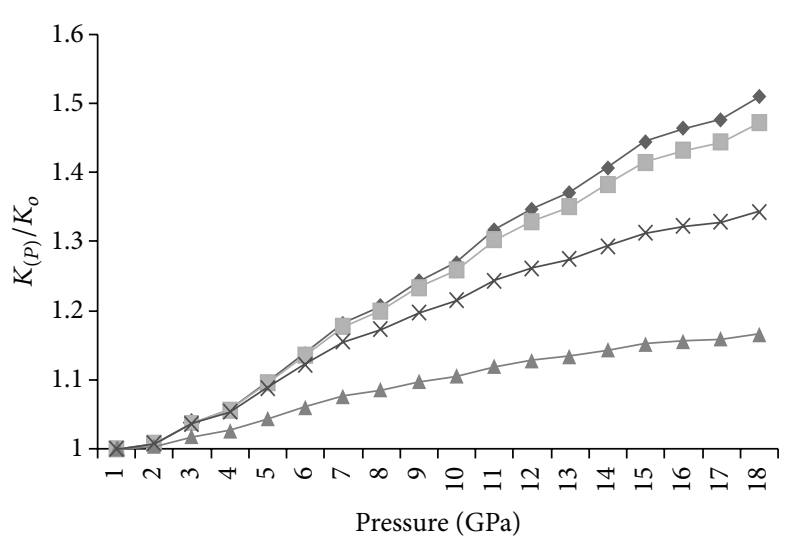

(b) $\mathrm{Ge}(13 \mathrm{~nm})$

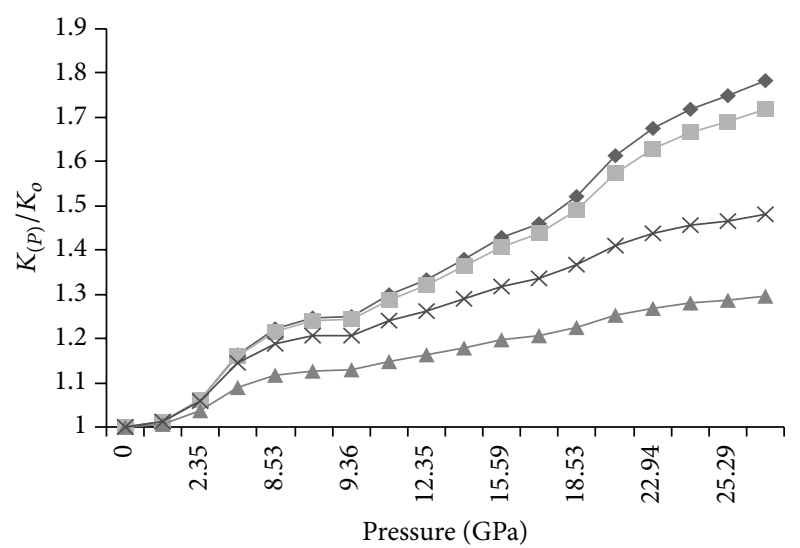

(d) Ni-filled MWCNT

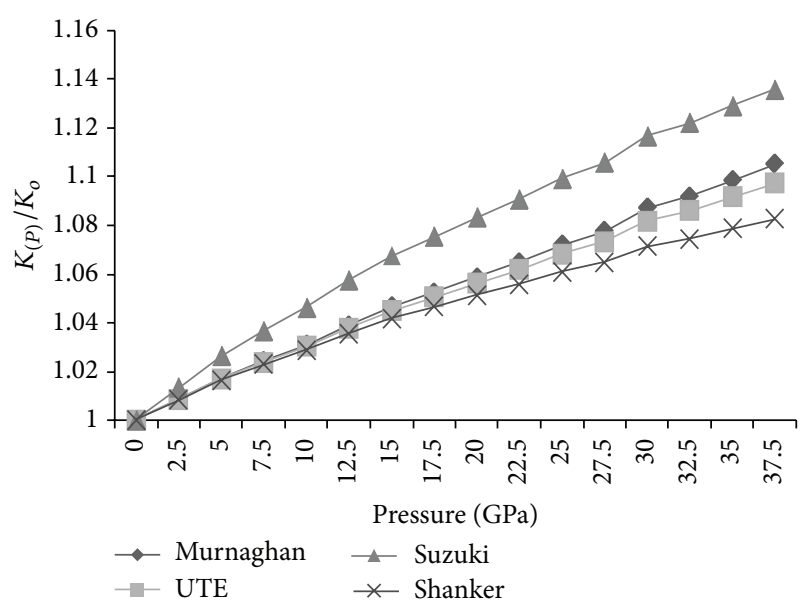

(f) $\mathrm{WC}(25 \mathrm{~nm})$

FIGURE 2: Variation of relative Bulk modulus $\left(K_{(P)} / K_{0}\right)$ with pressure $(P)$ for different materials.

used to calculate the relative volume compression $\left(V / V_{0}\right)$, isothermal bulk modulus $\left(K_{(P)} / K_{0}\right)$, and compression coefficient $\left(\alpha_{(P)} / \alpha_{0}\right)$ for the above nanomaterials are reported in Table 1 with their corresponding references.

The application of these equations of state is fully tested by calculating the mechanical properties of nanomaterials cited previously. The high pressure compression in the nanomaterials is calculated by using isothermal equations (2), (6), (19), and (25) relative bulk modulus and relative isothermal compression coefficient at different pressure is calculated by using (3), (7), (20), (26), (4), (9), (21), and (27), respectively.

The calculated results for all the nanomaterials have been shown in graphical form to show the validity of used equations of state with available experimental data. Figures 1(a) to 1 (f) show the relative compression with pressure for different materials, Figures 2(a) to 2(f) show the result of relative bulk modulus, and Figures $3(\mathrm{a})$ to $3(\mathrm{f})$ represents the result of relative compression coefficient. The error bar 


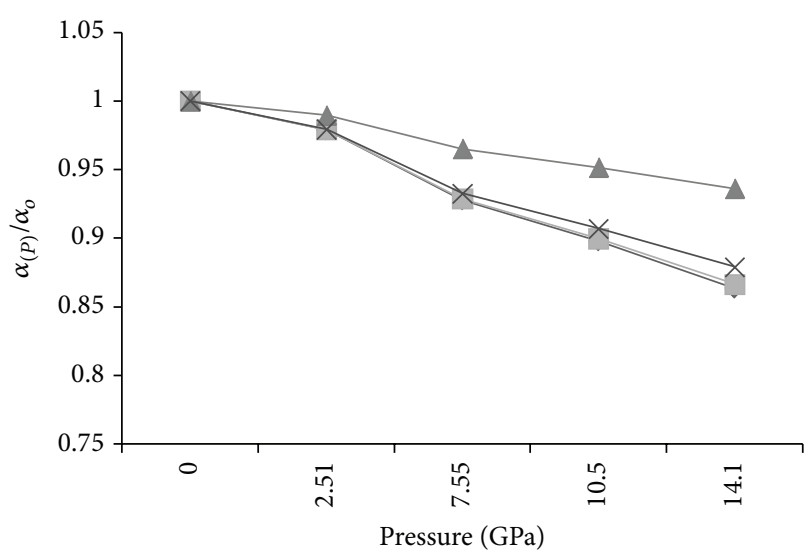

(a) $\operatorname{AlN}(10 \mathrm{~nm})$

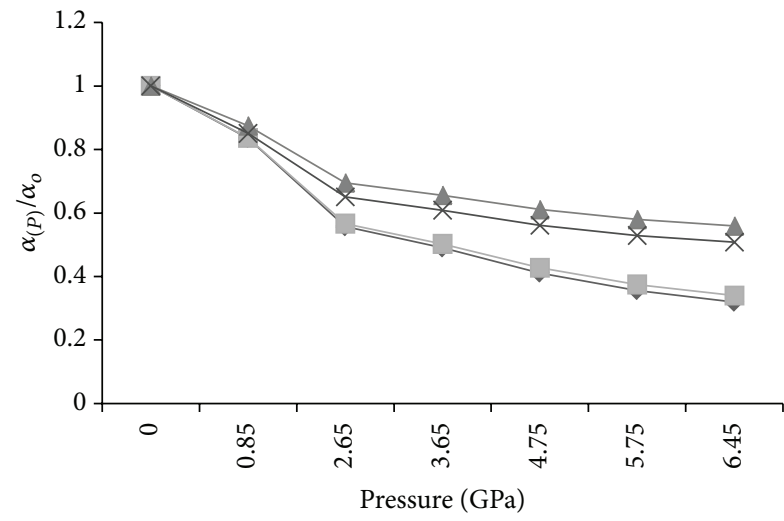

(c) $\mathrm{CdSe}(5.2 \mathrm{~nm})$

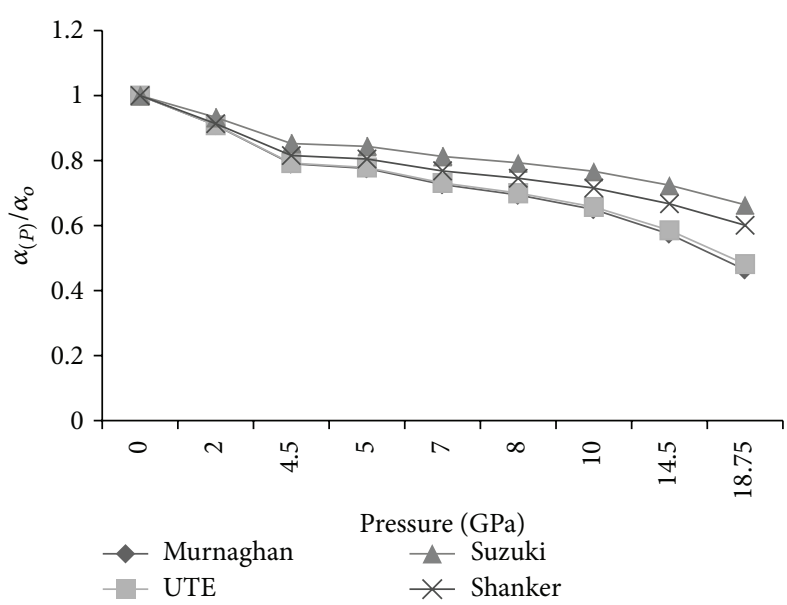

(e) Fe-filled MWCNT

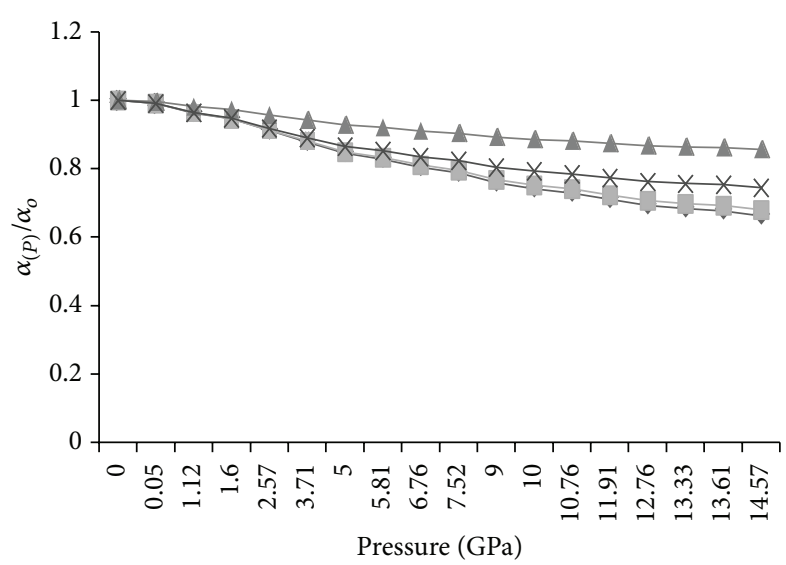

(b) $\mathrm{Ge}(13 \mathrm{~nm})$

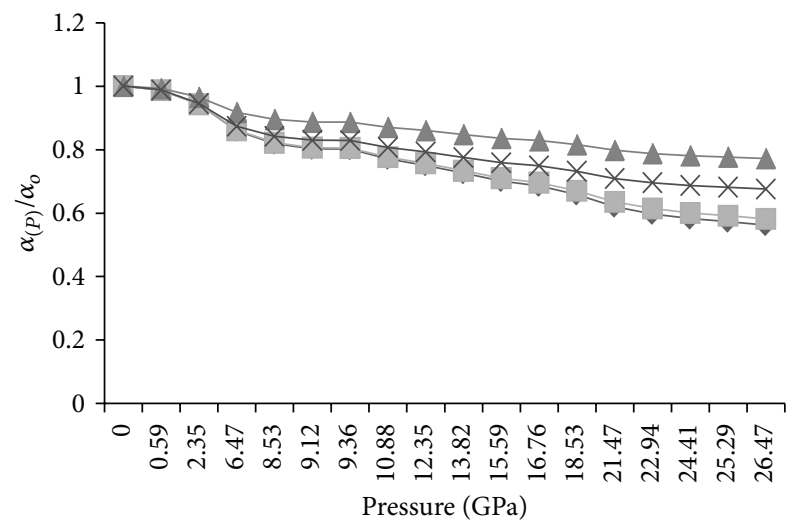

(d) Ni-filled MWCNT

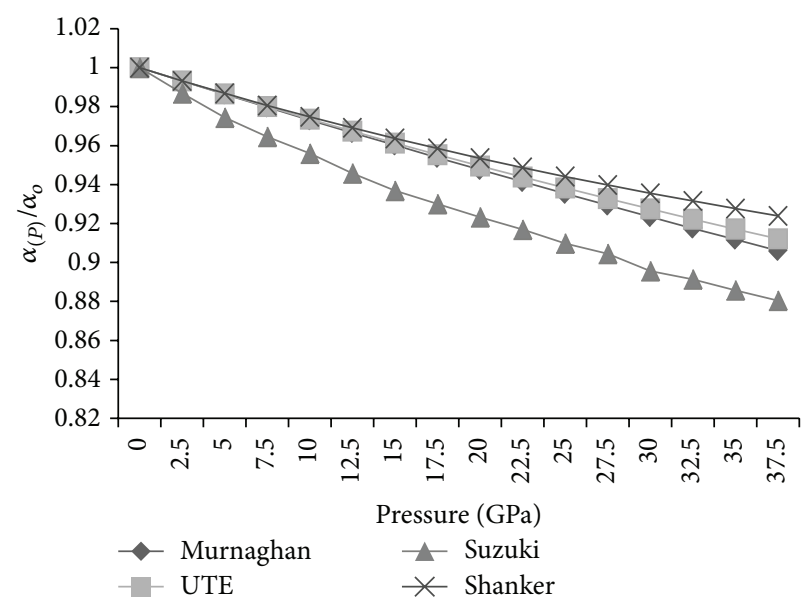

(f) WC $(25 \mathrm{~nm})$

FIGURE 3: Variation of relative compression coefficient $\left(\alpha_{(P)} / \alpha_{0}\right)$ with pressure $(P)$ for different materials.

diagrams are shown in Figures 4(a) to 4(f) for the sake of comparison.

\section{Discussion}

From graphical representation (Figures 1(a) to 1(f)), we see that the $V / V_{0}$ decreases with the increase in pressure $P$. We have observed that all four formulations are in close agreement with their experimental values for all nanomaterials at lower pressure range. Error bar diagrams from Figures 4(a) to 4(f) also explain this where E1, E2, E3, and E4 show the deviation of theoretical values from experimental data which are calculated by Murnaghan, UTE, Suzuki, and Shanker EOS, respectively. This shows the validity of all these equations of state in nanomaterials at lower pressure 


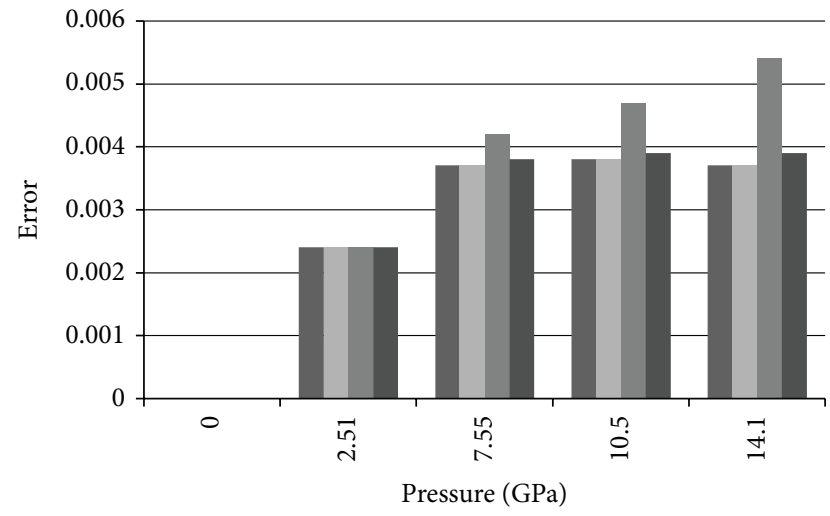

(a) $\operatorname{AlN}(10 \mathrm{~nm})$

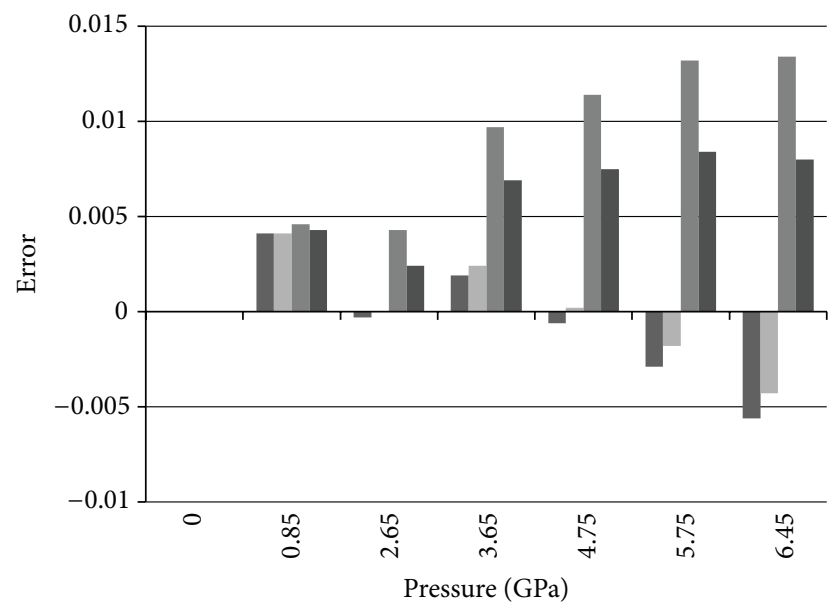

(c) $\mathrm{CdSe}(5.2 \mathrm{~nm})$

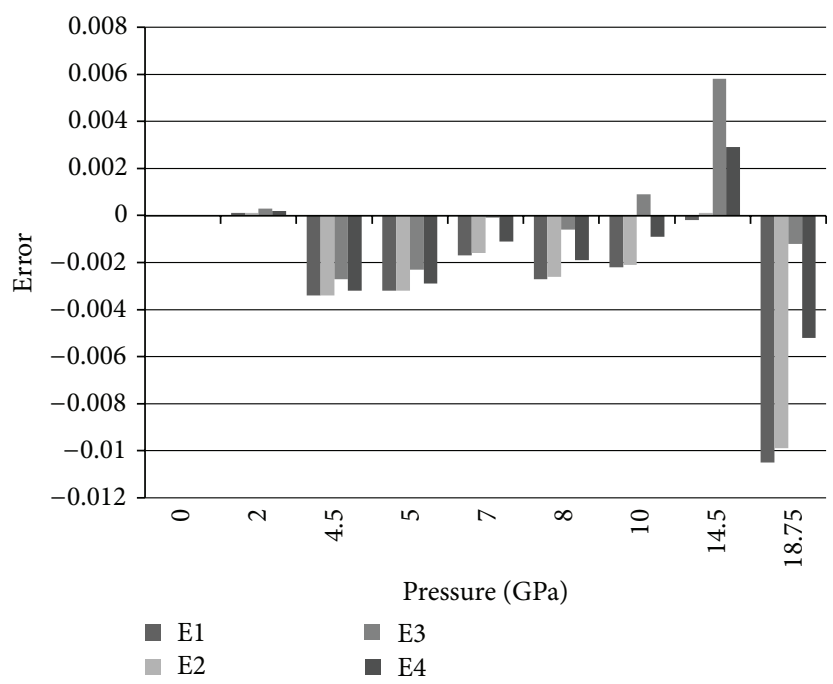

(e) Fe-filled MWCNT

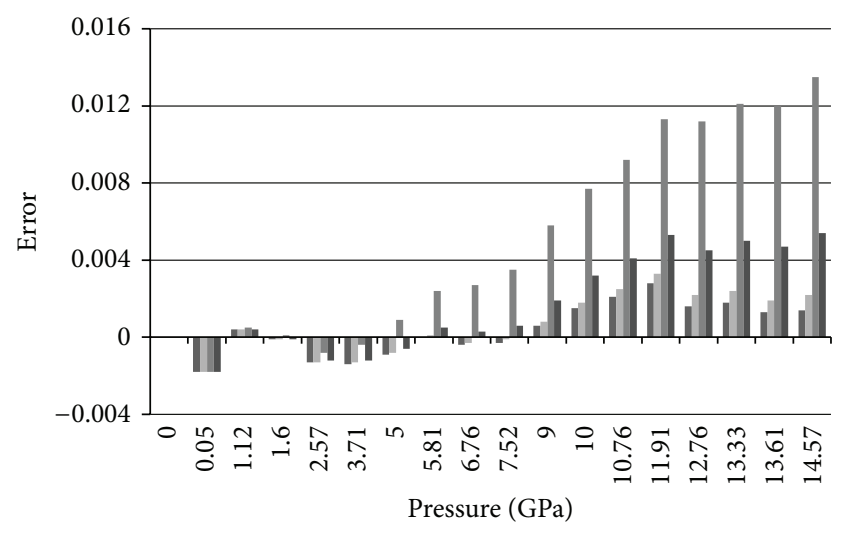

(b) $\mathrm{Ge}(13 \mathrm{~nm})$

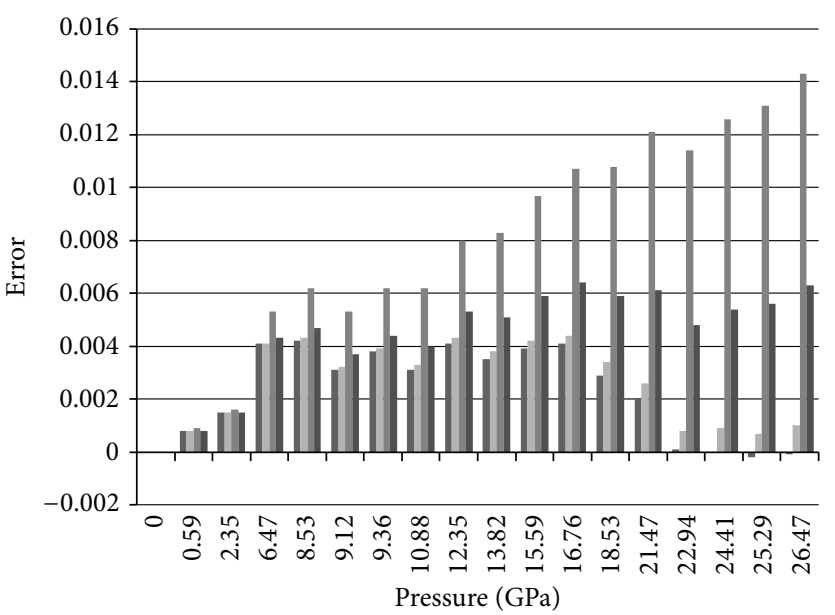

(d) Ni-filled MWCNT

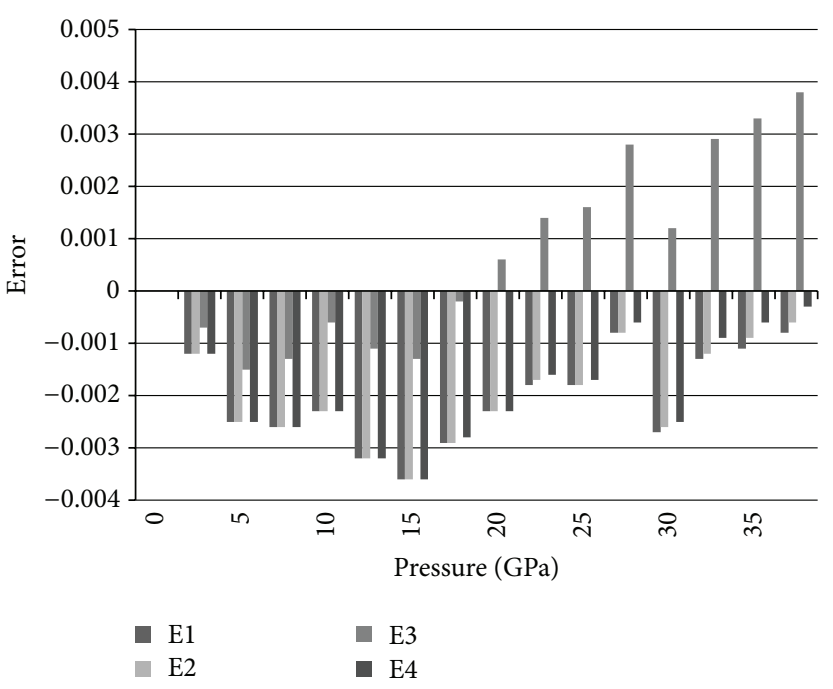

(f) $\mathrm{WC}(25 \mathrm{~nm})$

FIGURE 4: Error ( $Y$-axis) between experimental and calculated relative volume compression $\left(V / V_{0}\right)$ at various pressures $(X$-axis) for different materials. 
TABLE 1: Input parameters for different nanomaterials at reference temperature and zero pressure. Bulk modulus $K_{0}$ and first order pressure derivative of bulk modulus $K_{0}^{\prime}$ are taken from the references which are given in square brackets.

\begin{tabular}{lcc}
\hline Material & $K_{0}(\mathrm{GPa})$ & $K_{0}^{\prime}$ \\
\hline AlN $(10 \mathrm{~nm})$ & $321[5]$ & $4[5]$ \\
Ge $(13 \mathrm{~nm})$ & $112[3]$ & $4[3]$ \\
CdSe $(5.4 \mathrm{~nm})$ & $37[4]$ & $11[4]$ \\
Ni-filled MWCNT & $179.8[8]$ & $5.3[8]$ \\
Fe-filled MWCNT & $167[8]$ & $8.5[8]$ \\
WC $(25 \mathrm{~nm})$ & $416[7]$ & $4[7]$ \\
\hline
\end{tabular}

range and shows that different materials are compressible up to different extent. At higher pressure range, Murnaghan equation of state shows the best result; however, the usual Tait EOS is also near to experimental values but data obtained by Shanker and Suzuki formulations show more deviation.

It is clear that variations of relative isothermal bulk modulus increase with the increase of pressure and variations of relative compression coefficient decrease with the increase of pressure. It may be thus concluded that all these equations explain the compression behaviour of nanomaterials satisfactorily for lower pressure range. From Figures 2(a) to 2(f) and Figures 3(a) to 3(f), we observe that at high pressure results of Murnaghan and UTE coincide well and Shanker gives somewhat closer results to Murnaghan and UTE but Suzuki deviates.

The variation of relative isothermal bulk modulus and relative isothermal compression coefficient with pressure could not be compared with the experimental values since the experimental data on these physical properties of nanomaterials are under study and not are available so far.

\section{Conclusion}

Thus, it is clear that in all formulations there is an increment in the ratio of isothermal bulk modulus with pressure while relative isothermal compression decreases with increasing pressure and different material is compressible up to different levels. With the increase in pressures solid becomes more rigid. This is well understood as at high pressure, molecules come closer and their electron clouds ripple each other. The present study shows that all these equations of state are valid for nanomaterials at lower pressure range. It needs some modification at high pressure range for nanomaterials.

\section{References}

[1] J. Z. Jiang, "Phase transformations in nanocrystals," Journal of Materials Science, vol. 39, no. 16-17, pp. 5103-5110, 2004.

[2] S. Ramasamy, D. J. Smith, P. Thangadurai et al., "Recent study of nanomaterials prepared by inert gas condensation using ultra high vacuum chamber," Pramana, vol. 65, no. 5, pp. 881-891, 2005.

[3] H. Wang, J. F. Liu, Y. He et al., "High-pressure structural behaviour of nanocrystalline Ge," Journal of Physics: Condensed Matter, vol. 19, no. 15, Article ID 156217, 10 pages, 2007.
[4] S. H. Tolbert and A. P. Alivisatos, "The wurtzite to rock salt structural transformation in CdSe nanocrystals under high pressure," Journal of Chemical Physics, vol. 102, no. 11, article 4642, 15 pages, 1994.

[5] Z. Wang, K. Tait, Y. Zhao et al., "Size-induced reduction of transition pressure and enhancement of bulk modulus of AlN nanocrystals," Journal of Physical Chemistry B, vol. 108, no. 31, pp. 11506-11508, 2004.

[6] G. M. Amulele, M. H. Manghnani, S. Marriappan et al., "Compression behavior of WC and WC-6\%Co up to $50 \mathrm{GPa}$ determined by synchrotron x-ray diffraction and ultrasonic techniques," Journal of Applied Physics, vol. 103, no. 11, Article ID 113522, 6 pages, 2008.

[7] Z. Lin, L. Wang, J. Zhang, H. Mao, and Y. Zhao, "Nanocrystalline tungsten carbide: as incompressible as diamond," Applied Physics Letters, vol. 95, no. 21, Article ID 211906, 3 pages, 2009.

[8] H. K. Poswal, S. Karmakar, P. K. Tyagi et al., "High-pressure behavior of Ni-filled and Fe-filled multiwalled carbon nanotubes," Physica Status Solidi (b), vol. 244, no. 10, pp. 3612-3619, 2007.

[9] O. L. Anderson, "The use of ultrasonic measurements under modest pressure to estimate compression at high pressure," Journal of Physics and Chemistry of Solids, vol. 27, no. 3, pp. 547$565,1966$.

[10] S. H. Tolbert and A. P. Alivisatos, "Size dependence of a first order solid-solid phase transition: the wurtzite to rock salt transformation in CdSe nanocrystals," Science, vol. 265, no. 5170, pp. 373-376, 1994.

[11] S. H. Tolbert and A. P. Alivisatos, "High-pressure structural transformations in semiconductor nanocrystals," Annual Review of Physical Chemistry, vol. 46, pp. 595-626, 1995.

[12] J. Z. Jiang, J. S. Olsen, L. Gerward, and S. Morup, "Enhanced bulk modulus and reduced transition pressure in $\gamma-\mathrm{Fe}_{2} \mathrm{O}_{3}$ nanocrystals," Europhysics Letters, vol. 44, no. 5, article 620, 1998.

[13] J. Z. Jiang, L. Gerward, D. Frost, R. Secco, J. Peyronneau, and J. S. Olsen, "Grain-size effect on pressure-induced semiconductorto-metal transition in ZnS," Journal of Applied Physics, vol. 86, no. 11, pp. 6608-6610, 1999.

[14] J. Z. Jiang and L. Gerward, "Phase transformation and conductivity in nanocrystal $\mathrm{PbS}$ under pressure," Journal of Applied Physics, vol. 87, no. 5, article 2658, 3 pages, 2000.

[15] B. Chen, D. Penwell, L. R. Benedetti, R. Jeanloz, and M. B. Kruger, "Particle-size effect on the compressibility of nanocrystalline alumina," Physical Review B, vol. 66, no. 14, Article ID 144101, 4 pages, 2002.

[16] Y. He, J. F. Liu, W. Chen et al., "High-pressure behavior of $\mathrm{SnO}_{2}$ nanocrystals," Physical Review B, vol. 72, no. 21, Article ID 212102, 4 pages, 2005.

[17] Z. Wang, L. L. Daemen, Y. Zhao et al., "Morphology-tuned wurtzite-type ZnS nanobelts," Nature Materials, vol. 4, pp. 922927, 2005.

[18] A. San-Miguel, "Nanomaterials under high-pressure," Chemical Society Reviews, vol. 35, no. 10, pp. 876-889, 2006.

[19] I. Suzuki, "Thermal expansion of periclase and olivine, and their anharmonic properties," Journal of Physics of the Earth, vol. 23, no. 2, pp. 145-159, 1975.

[20] I. Suzuki, K. Seya, H. Tokai, and Y. Sumino, "Thermal expansion of single-crystal manganosite," Journal of Physics of the Earth, vol. 23, no. 1, pp. 63-69, 1979. 
[21] F. D. Murnaghan, "The compressibility of media under extreme pressures," Proceedings of the National Academy of Sciences of the United States of America, vol. 30, no. 9, pp. 244-247, 1944.

[22] J. L. Tallon, "The thermodynamics of elastic deformation-I. Equation of state for solids," Journal of Physics and Chemistry of Solids, vol. 41, no. 8, pp. 837-850, 1980.

[23] F. Birch, "Equation of state and thermodynamic parameters of $\mathrm{NaCl}$ to $300 \mathrm{kbar}$ in the high-temperature domain," Journal of Geophysical Research, vol. 91, no. 5, pp. 4949-4954, 1986.

[24] J. Shanker and S. S. Kushwah, "Analysis of thermodynamic properties for high-temperature superconducting oxides," Physica Status Solidi (b), vol. 180, no. 1, pp. 183-188, 1993.

[25] H. Schlosser and J. Ferrante, "Liquid alkali metals: equation of state and reduced-pressure, bulk-modulus, sound-velocity, and specific-heat functions," Physical Review B, vol. 40, no. 9, pp. 6405-6408, 1989.

[26] M. Kumar, "High pressure equation of state for solids," Physica B, vol. 212, no. 4, pp. 391-394, 1995.

[27] M. Kumar, "Thermal expansivity and equation of state up to transition pressure and melting temperature: $\mathrm{NaCl}$ as an example," Solid State Communications, vol. 92, no. 5, pp. 463466, 1994

[28] J. R. Macdonald, "Some simple isothermal equations of state," Reviews of Modern Physics, vol. 38, no. 4, pp. 669-679, 1966.

[29] O. L. Anderson and D. Issak, "The dependence of the AndersonGrüneisen parameter $\delta_{T}$ upon compression at extreme conditions," Journal of Physics and Chemistry of Solids, vol. 54, no. 2, pp. 221-227, 1993.

[30] M. P. Toshi, "Cohesion of ionic solids in the born model," Solid State Physics, vol. 1, pp. 1-120, 1964.

[31] J. Shanker, B. Singh, and S. S. Kushwah, "On the high-pressure equation of state for solids," Physica B, vol. 229, no. 3-4, pp. 419420, 1997. 

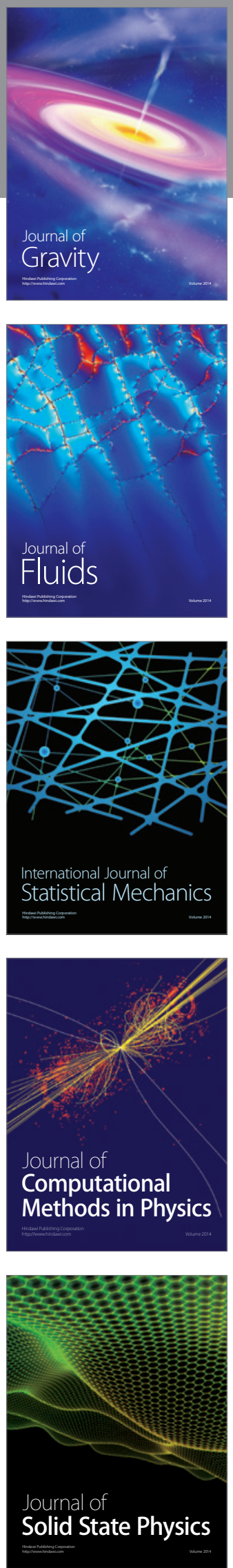

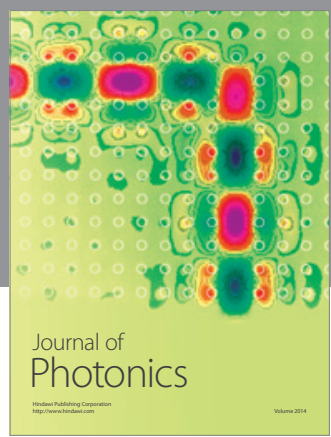

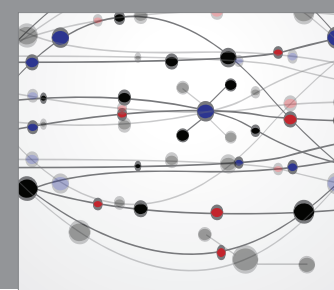

The Scientific World Journal

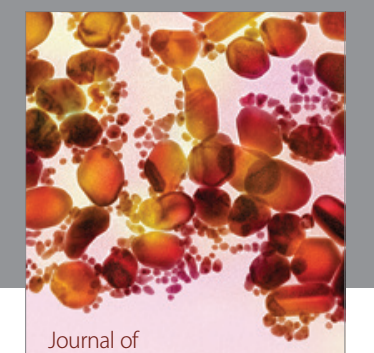

Soft Matter
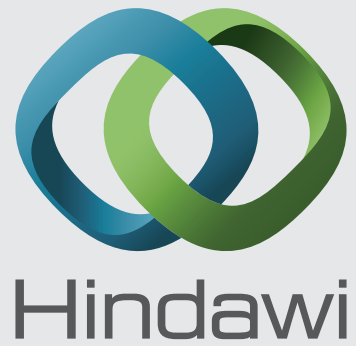

Submit your manuscripts at

http://www.hindawi.com
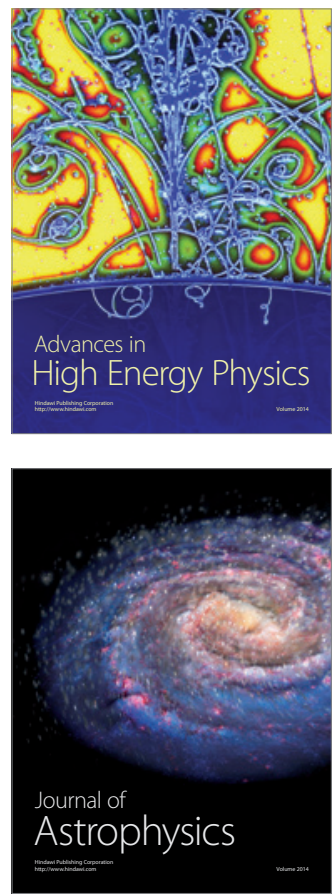
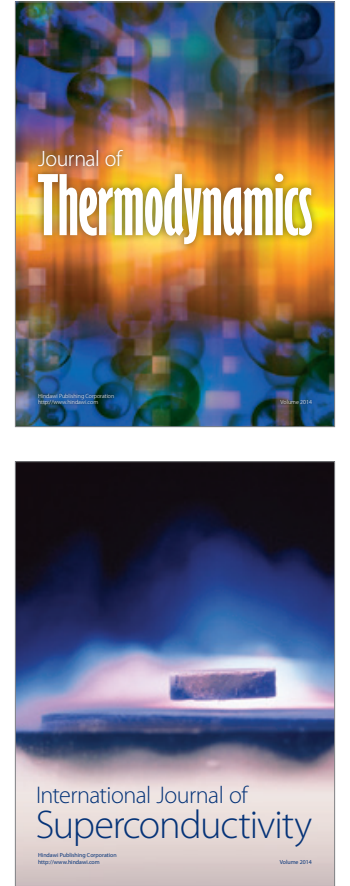
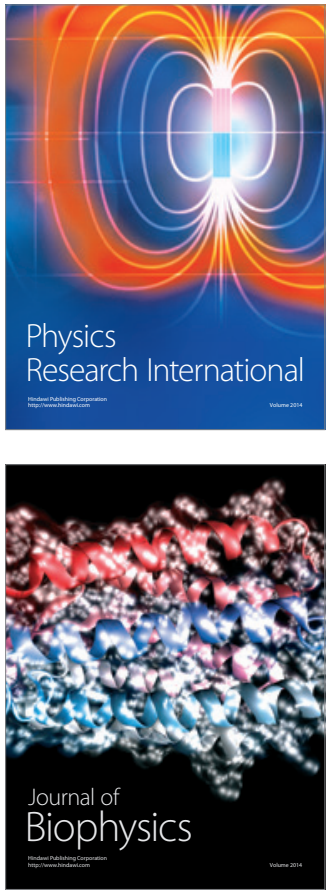
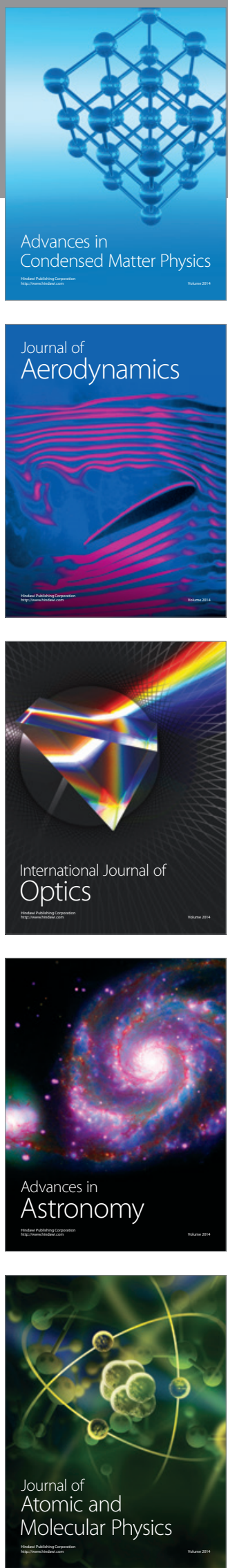